\title{
Design and Applications of Soft Sensors in Polymer Processing: A Review
}

DOI:

10.1109/jsen.2018.2885609

\section{Document Version}

Accepted author manuscript

Link to publication record in Manchester Research Explorer

\section{Citation for published version (APA):}

Abeykoon, Y. M. C. (2018). Design and Applications of Soft Sensors in Polymer Processing: A Review. Sensors Journal, IEEE, [10.1109/JSEN.2018.2885609]. https://doi.org/10.1109/jsen.2018.2885609

\section{Published in:}

Sensors Journal, IEEE

\section{Citing this paper}

Please note that where the full-text provided on Manchester Research Explorer is the Author Accepted Manuscript or Proof version this may differ from the final Published version. If citing, it is advised that you check and use the publisher's definitive version.

\section{General rights}

Copyright and moral rights for the publications made accessible in the Research Explorer are retained by the authors and/or other copyright owners and it is a condition of accessing publications that users recognise and abide by the legal requirements associated with these rights.

\section{Takedown policy}

If you believe that this document breaches copyright please refer to the University of Manchester's Takedown Procedures [http://man.ac.uk/04Y6Bo] or contact uml.scholarlycommunications@manchester.ac.uk providing relevant details, so we can investigate your claim.

\section{OPEN ACCESS}




\title{
Design and Applications of Soft Sensors in Polymer Processing: A Review
}

\author{
Chamil Abeykoon
}

\begin{abstract}
In manufacturing industry, process monitoring is a key to observe the product quality, operational health, safety and also for achieving good/satisfactory process control performance. In polymer processing, the level of control of the process operational quality is vastly dependent upon the performance of the process monitoring techniques as it is quite difficult to observe the inside of an extruder barrel (or processing chamber) during the process operation. Currently, a number of physical sensing devices/techniques are widely available for industrial applications for monitoring of parameters such as melt temperature, melt pressure, screw/processing speed, product dimensions, etc in real-time. However, there may be some limitations in using physical sensors in piratical process measurements (such as melt viscosity and melt temperature profile) due to several constraints such as their access requirements, disruptive effects to the melt flow, fragility, complexity, etc. Therefore, soft sensing techniques should be highly useful for improved process monitoring and modelling, and hence for advanced process control. In this work, a general description is presented on the state-of-the-art of soft sensor design. Then, a comprehensive review is made on the use of soft sensing techniques in polymer processing applications while identifying their capabilities and limitations. Eventually, the importance of developing of such soft sensing techniques (together with some sort of built-on intelligence) for the advancement of process monitoring is discussed while indicating some of the possible directions for future industry.
\end{abstract}

Index Terms-Process monitoring, Inferential measurements, Soft sensor, Intelligent systems, Modelling and Control, Melt temperature, Melt viscosity, Melt flow index, Torque, Polymer processing.

\section{INTRODUCTION}

Process monitoring is one of the important aspects in manufacturing industry as this information may be highly crucial in observing the process health and hence to maintain the process functionality within the desired limits. However, the direct use of physical sensors may not be possible in some particular applications, occasions or environments due to a number of constraints such as access requirements, disturbances to the process output/functionality, harshness of the environment, limitations of the sensors and so forth. Although some process parameters are really important in determining the process functional quality, they may not be monitored/measured in real-time by using a physical sensor. Therefore, as an alternative/extended approach of process monitoring, concepts of virtual instruments (VIs) and soft sensors became widely popular particularly over the last three decades [1], [2].

\section{A. Soft sensor}

The term 'soft sensor' can have different definitions where it is a widely-used name for software-based sensors where several measurements (may be just one, dozens or even hundreds

C. Abeykoon is with the School of Materials, Faculty of Science and Engineering, The University of Manchester, Oxford Road, Manchester, M13 9PL, United Kingdom, E-mail: chamil.abeykoon@manchester.ac.uk, yabeykoon01@qub.ac.uk of measurements) are processed together. Also the interaction of the signals can be used for calculating new quantities that cannot be measured in practice. Hence, the term soft sensor is based on the words 'software' and 'sensor'. To be compatible with this paper, a general definition can be given as: a soft sensor (also known as a virtual sensor or an inferential estimator) is a technique of estimating process parameter/s (e.g., qualitybased measures, variables indicating functionality, faults) in various applications when a hardware sensor is unavailable or unsuitable for making direct measurements. Generally, such soft/inferential sensors are used in real-time process monitoring, modelling and control; fault detection/estimation; process diagnostics, etc mostly in continuous processes. Currently, these sensors are widely used in many industrial applications such as polymerization reactors, distillation columns, cement kilns, food processing, paper/pulp production industry, etc, to estimate the product quality parameters [3]. Obviously, soft sensors can be continuous sources of information on process functionality, product quality and so forth. Furthermore, these can be involved in automated process control loops as well and hence can provide some benefits such as increased effectiveness of the process, optimum energy usage, improved safety and troubleshooting, etc [4]. In general, soft sensors can be classified into two types: 'model-driven' which are based on the first principle models and 'data-driven' which are based on the actual data measured from the processing plants. Of these two types, model-driven soft sensors usually focus on the ideal steady-state of the processes (physical/chemical background of the process) while the data-driven soft sensors can have good potential of representing real process dynamics. Hence, the data-driven approaches are quite popular in the process industry although model-driven approaches may also be important depending on the type of the process. Generally, there are a number of issues/challenges that should be dealt with in developing both types.

In most of the previously reported soft sensing applications, the behaviours (i.e., mostly non-liner) of the industrial processes have been modelled with the techniques such as principle component regression (PCR), artificial neural network (ANN); fuzzy and/or neuro-fuzzy systems; partial least squares (PLS); support vector machine (SVM); radial basis function (RBF) neural networks and support vector regression (SVR), and most of these will be discussed in the next section. The popularity of these techniques is particularly due to their flexibility of modifying into adapting or evolving methods which are capable in reacting to changes in the data and thus to learn knowledge base, if necessary. More details on each of these soft sensor development methods and comparisons of these methods can be found in the literature [2], [5]-[7], and hence not presented in this paper. 


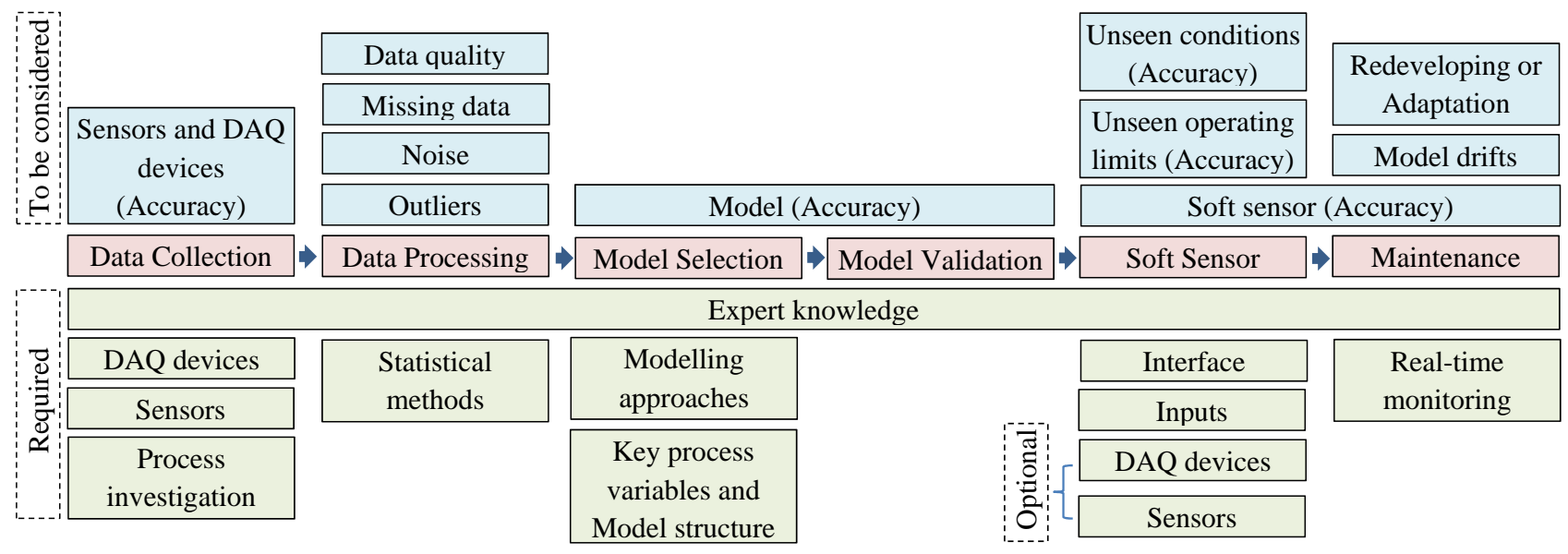

Figure 1. Basic steps for designing of a soft sensor

Some of the major steps in designing of a soft sensor are shown in Figure 1. With the rapid development of computational facilities/technologies over the last few decades, soft sensing approaches are becoming widely popular in various industrial and remote applications due to their advantages such as [8]:

- the easiness of implementing on the existing hardware platforms (no or little additional investment may be required). - the easiness of updating the facilities (extensions or taking parts off) as required.

- the usefulness in the applications which hardware/physical sensors may not be applicable or unsuitable (or when they are too expensive) for making direct measurements.

- the possibility of providing real-time estimations while taking time delays in to account.

- the possible easiness and simplicity of maintenance.

- the possibility of using as a low cost alternative for expensive commercial online analyser tools.

On the other hand, design/application of the soft sensors might be quite challenging due to a few reasons [8]:

- Obviously, a considerable expert knowledge (on both industrial process and the design procedure of the sensor), effort and time should be required to design a soft sensor.

- Their performance usually depend on the quality of the training/validation data while some issues can be arise due to outliers, noise and missing data, etc.

- Data-driven soft sensors may be specific mostly for a given material, machine or processing conditions used to collect the test data. Use of generalized process models would be one of the best possible options to tackle this issue.

As was mentioned, the designers of soft sensors should have a sound knowledge on all the areas presented in Figure 1 where they have to spend lots of time in data collection and processing (dealing with missing values, outliers, etc) and model training/validation/optimization. Also, they should have some knowledge on the related industrial process/application, otherwise can consult a process related expert during the design. Further details on the soft sensor design and adaptation can be found in the published literature [2], [7]-[20].

\section{B. Polymer processing}

Polymers are one of the popular materials in the modern world where the world total production of plastics has been reported as 256 million tonnes in 2010. In other words, the annual average global consumption of plastics per head has increased from $10 \mathrm{~kg}$ in 1980 to $40 \mathrm{~kg}$ in 2010 marking a drastic growth rate of consumption [21]. It is predicted that this growth may steady or further increase in next few decades due to the superior properties of polymeric or polymer matrix based composite materials such as high specific strength that is highly desirable in harmful emission control particularly in the transportation sector. Perhaps, plastics/polymers might be the most widely used materials in the present world and also have been named as the 'the materials for the $21 \mathrm{st}$ century' [22], [23]. A number of different polymer processing techniques are used in the current industry based on the nature of the application, product, material, and so forth: Extrusion and Extrusion-based processes, Injection moulding, Thermoforming, Compression moulding, Rotational moulding, Blow moulding, etc. More details on the mechanisms, theories and functional requirements of polymer processing are available in the literature [24]-[30].

\section{SOFT SENSING OF IMPORTANT PROCESS PARAMETERS IN POLYMER PROCESSING}

In polymer processing, it is highly difficult to observe/monitor the process melt quality as the material is being processed inside a closed barrel (for example: an extruder is a kind of black box) [31]. In extrusion or in injection moulding, after feeding the material through the hopper, material absorbs the heat while conveying along a screw and forms into the desired shape inside a die/mould which is almost a closed process. In other polymer processing techniques also materials are usually processed inside a closed chamber limiting the process diagnostics. In these processes, the quality of the process melt output has to be determined mostly observing/testing the quality of the final product and then it may be too late to take any 
precautions if there is any issue. While processing parameters such as melt pressure and melt temperature (point and/or bulk measurements) can be observed in real-time using physical sensors, some important process parameters such as the die melt temperature profile, melt viscosity and melt flow index which are really important to determine the process functional quality and also the quality of the melt output, are quite difficult to measure physically. No sensors are commercially available yet to measure most of these parameters in realtime, to the author's knowledge. Therefore, further research and development on inferential monitoring of these parameters would be promising alternatives for the current/future industry.

\section{A. Soft sensing of die melt temperature profile}

The thermal homogeneity of the melt output is a key expectation in all types of polymer processing applications and hence the melt temperature is one of the commonly measured parameters in these processes. A number of different types of thermal monitoring techniques are used in the current polymer processing industry and these details were discussed by the author previously [28], [32], [33]. At present, point and/or bulk thermal monitoring techniques are well-established and are widely used in industrial applications [27], [34], [35]. However, these are not capable of detecting thermal variations across the melt flow and can only provide some limited amount of information close to where they are located. In fact, the measurement of a melt temperature profile is highly suitable for determining the actual thermal homogeneity and stability of the melt output of any polymer processing technique. Some of the temperature measurement techniques used in research (e.g., a fluorescence technique [36], a thermocouple mesh [37]) are capable of measuring a temperature profile across the melt flow but these techniques are not yet suitable for use in an industrial applications due to constraints such as their limited durability, access requirements, complexity, disruptive effects on melt flow and output, etc. Thus, soft sensing approaches for die melt temperature profile should be highly useful.

As was discussed above, monitoring of a melt temperature profile (in real-time) is quite problematic in industrial polymer processing applications with physical sensors. The first soft sensing concept to predict a temperature profile of an extruder's output melt flow was proposed by the author recently [8], [14], [38], [39] and the structure of this conceptual design is shown in Figure 2. Here, $T_{m, j}$ is the temperature of a given point across the melt flow which is $j \mathrm{~mm}$ away from the melt flow centreline, $\omega_{s c}$ is the processing screw rotational speed, $T_{b}$ is the set temperatures along the extruder barrel where subscript $i$ represents the number set temperature zones: $T_{b, 1}, T_{b, 2}, \cdots, T_{b, n}$ and $R_{p, j}$ is the die radial position.

Basically, this melt temperature profile predictive soft sensor can be implemented only based on a few readily measurable parameters (screw speed, barrel set temperatures, and a melt temperature measurement from a flush mounted infrared (IR) temperature sensor) of a polymer extrusion process. Mainly, the proposed soft sensor operates based on two data-driven dynamic models. One model predicts the melt temperature at

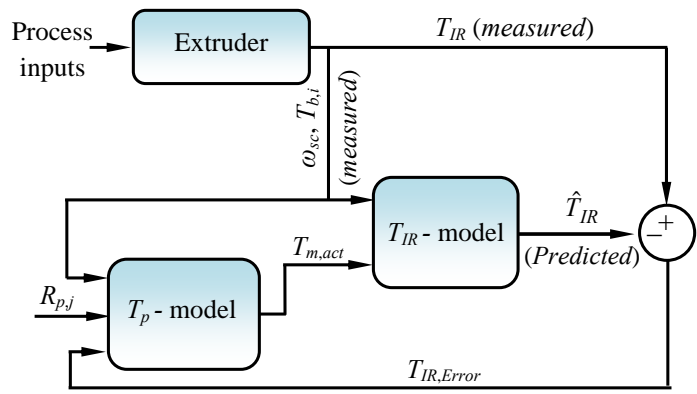

Figure 2. A schematic of the structure of the proposed extruder die melt temperature profile predictive soft sensor concept [14]

different radial positions across the melt flow (i.e., the $T_{P}$ model). The other model is to predict the melt temperature given by an IR temperature sensor (i.e., the $T_{I R}$ or feedback model) and this is based on the predictions of the $T_{P}$ model as well. This soft sensor should be really useful for improved process monitoring and also to use as a mode of feedback for process control, if it can be improved for industrial use. To the author's knowledge, no any other work has been reported on soft sensing of a temperature profile across the output melt flow of any polymer processing technique.

\section{B. Soft sensing of melt viscosity}

Viscosity is one of the key indicators of process melt quality. Bruss [40] argues that melt viscosity is a much more suitable control variable than temperature or pressure in polymer processing. However, it is not directly measurable or controllable and can only be affected by other process variables such as processing speed and set process temperatures. Consistency of the melt viscosity is highly important for good product quality and hence avoiding defects. Therefore, accurate viscosity measurements are highly desirable in polymer processing applications. A number of previous researchers attempted to measure the melt viscosity by using physical sensors [41] [45]. Of these physical viscosity monitoring techniques, offline methods are attributed with considerable inaccuracies as measurements are made away from the actual processing plant/unit. Side stream methods are carried out on the machine but suffer from delays due to time taken to the melt to reach the measuring device. Also, off-line and side stream methods are shown to be inappropriate for accurate detection of process dynamics. In-line devices eliminate time delays associated with side stream devices and may detect process dynamics as well. Unfortunately, there are practical limitations of using inline devices in industry due to their possible flow restrictions. Flow restrictions can lead to several adverse issues such as product defects, higher process pressures, higher energy consumption and reduced productions rates, etc.

Since physical viscosity measurements are difficult, soft sensing techniques would be promising. McAfee et al. [46], [47] proposed a model-based soft approach which includes two data-driven models to estimate the melt viscosity of an extrusion process and the structure is shown in Figure 3. The first model predicts the melt viscosity based on a few 


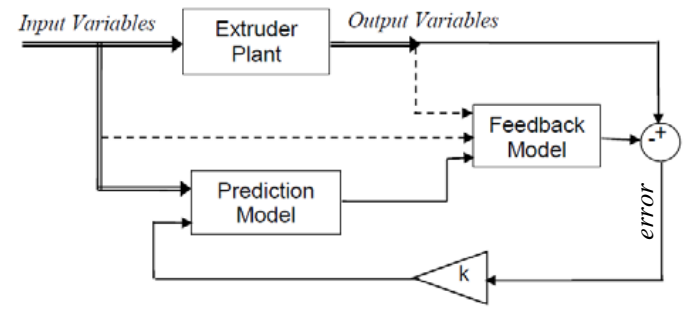

Figure 3. The structure of the viscosity soft sensor proposed by McAfee et al. [46], [47]

readily measurable process parameters (i.e., screw speed and set temperatures) and while the second model predicts the melt pressure based on the estimated melt viscosity. Then, the actually measured melt pressure is compared with the estimated pressure, both at the die, and the error signal (i.e., the mismatch between pressures) is used to compensate for possible errors in the melt viscosity prediction model. Sensor's dynamic models were developed using a grey box modelling approach and it gave predictions within $2 \%$ error. This would be a good inferential real-time viscosity sensor if it can be improved for industrial use.

Chen et al. [48] suggested an empirical model to evaluate the polymer melt viscosity and this model was used for quality control purposes in extrusion. The model calculates the melt viscosity based on experimental measurements of screw speed, melt temperature, geometrical dimensions of the screw (e.g. diameter, channel depth and helix angle) and material constants. The results were in agreement with the measurements obtained from an in-line rheometer. However, the model accuracy depends on the consistency of melt properties and it is not capable of indicating any fluctuations in the process.

Work by Kumar et al. [49] suggested an inferential approach for on-line estimation of melt viscosity and then this was used with a closed-loop control of a polymer compounding process which involves blending of two polymers. Moreover, a physics-based dynamic model was formulated to describe the dynamic effects of common process inputs (e.g., raw material feed rates, screw speed) on the measured outputs (e.g. die pressure). This dynamic model was coupled with on-line melt viscosity estimation to cover wide range of operating conditions including raw materials and composition changes. The accuracy of melt viscosity estimation is dependent upon the accuracy of the on-line measurements of feed rates and die pressure. The results showed that the estimated viscosity and the off-line measurements made by a capillary rheometer were matched only with $\pm 10 \%$ difference.

Gonzaga et al. [50] proposed a melt viscosity prediction soft sensor for a polyethylene therephthalate (PET) production process. It was based on a feed-forward artificial neural network and its predictions showed good accuracy only with a relative error of approximately $0.3 \%$. Moreover, this sensor was integrated with an industrial process control system through a supervisory system and the authors claimed that the controller (i.e., based on the viscosity soft sensor) gave good performance by allowing an effective and feasible operation of the PET production plant.

Work presented by Yaoguang et al [51] developed a datadriven soft sensor based on back-propagation neural networks with the aim of the viscosity control of the feed liquid in the production of PVC gloves. The sensor was able to predict the viscosity with a prediction error of $\pm 0.03 \%$. Moreover the authors claimed that other variables such as temperature and rate of production should also be considered along with the viscosity for achieving the better process control.

Kugler et al [52] proposed soft sensing models for melt viscosity of an extrusion process incorporating artificial neural networks. The data has been collected from the system identification experiments and then a software called NeuroModel was used to carry out the modelling. The soft sensor was tested with a few materials with different molecular structure where the measured and predicted viscosity values showed only a 0.03 difference of the coefficient of determination.

Abdallah et al [53] used a specially designed pressurewave-based acoustic sensor attached to a slit die for inline monitoring of polymer flow behavior. A correlation was observed between the measured resonance parameters and the fluid properties. Then, a boundary reflection based acoustic measurement approach was used to predict the viscosity of the melt. As their research was still in early stage they did not comment on the accuracy of the predictions. However, this will be promising for industrial use if it can be improved to be used with industrially usable die geometries, as this is an invasive method of predicting polymer properties in real-time.

Kazmer et al [54] proposed a multivariate sensor (MVS), based on a number of mechanistic relations, which can estimate the melt viscosity and velocity for determining the process state. This sensor is a combination of both soft and hardware aspects where a piezoelectric ring is used to monitor the melt pressure while a thermopile (a set of thermocouples that are pre-arranged to measure the radiant heat) is employed in acquiring the melt and mould temperatures. The sensor performance has been evaluated experimentally where it outperformed some of the commercially available sensors. The authors claimed that this MVS should be really useful in monitoring the melt consistency inside mould cavities of injection moulding and also in feedback control of the melt temperature, melt pressure and process timings.

Few other works [44], [45], [55]-[57] also considered the melt viscosity with the purpose of process control (i.e., to reduce the melt viscosity variations during polymer processing) and the viscosity prediction was not the major purpose of their research and hence not discussed in detail. As it is clear, no many works have been reported on soft sensing of melt viscosity and hence further research is highly recommended in this area.

\section{Soft sensing of melt flow index}

The melt flow index (MFI) is a type of measure which indicates the ease of flow of polymer melts. Currently, there is no good industrially compatible real-time MFI measurement 
method presented although several well-established off-line techniques are available [58].

In late 1990s, Ogawa et al. [59] reported a MFI soft sensor for an industrial high density polyethylene (HDPE) production process incorporating a few on-line measurements. Within this soft approach, two models were used: (i) an MFI model which describes a relationship between the MFI and other process variables (ii) a cumulative model that maps a relationship between the MFI of the total polymer inside the reactor. Then, the proposed MFI soft sensor was integrated with a model predictive controller dedicated to product quality control . As was claimed by the authors, the control system incorporated with the MFI soft sensor performed well with less product quality deviations but further testing should be required prior to using this sensor in industrial applications.

In 2006, Sharmin et al. [60] developed a partial least squares (PLS) based soft sensing approach to predict the MFI of polymers. The PLS soft sensing model was developed incorporating 48 process variables together with the corresponding time delays. Then, the sensor was implemented and tested on an industrial polymerization reactor and the authors claimed that successful results were achieved. Furthermore, they have made a discussion on possible challenges/difficulties associated in developing data-driven models from the data collected from industrial processes.

Work presented by Shi and Liu [61] developed a MFI estimation soft sensor for an industrial polypropylene (PP) polymerization plant. The soft sensing model was developed by a weighted least squares support vector machines (weighted LS-SVM) approach and it used nine process variables as inputs to predict the MFI. Moreover, this model performed well by predicting the MFI with a mean relative error (MRE) of $3.27 \%$ and outperformed the sensors based on LS-SVM and SVM approaches. Another work by Shi et al [62], [63] reported a product quality prediction soft sensor model based on a principal component analysis (PCA), a multi-scale analysis (MSA) and a radial basis function (RBF). The most relevant process parameters were identified by the PCA and the MFI was used as the quality measure, and the soft sensor was tested on the same industrial PP plant. With all the processing conditions tested, the PCA-MS-RBF model was able to estimate the MFI with a mean relative error of $3 \%$ while the average errors of $5 \%$ and $4 \%$ were obtained respectively from the MS-RBF and PCA-RBF models. Another subsequent work by $\mathrm{Li}$ and Liu [64] proposed a MFI soft sensing approach for the same PP polymerization plant based on a RBF neural networks incorporating an adaptive ant colony optimization algorithm. The authors claimed that their latest soft sensor was capable of predicting the MFI with an MRE of $0.44 \%$ which was superior in performance to the sensors proposed by their previous works. Moreover, they believe that their new MFI soft sensor shows a promising outlook in practical use.

Work presented by Ahmed et al [65], an empirical model was proposed to predict MFI in grade-changing HDPE polymerization plant. The nonlinearities of the polymerization process were captured by using a recursive partial least squares
(RPLS) algorithm and a model bias update. Two schemes (scheme I: a PLS algorithm + a constant bias; scheme II: a PLS algorithm + a range of bias with upper and lower limits) were developed and achieved RMSE values of 0.0941 and 0.0944 respectively on a set of unseen data. However, this work is based only on simulation.

Balask et al [66] also proposed a soft sensor to observe the product quality by an on-line MFI prediction of an industrial polymerization plant. A self-organizing map was used for the visual understanding of processes so that several variables and their interactions may be inspected simultaneously. This concept is based on a bank of historical process data stored and soft sensors which analyze the effect of particular transition trajectories on the MFI and hence the product quality. As claimed by the authors, this method is useful in several process related aspects such as analyzing the system performance, process optimization, reverse engineering, etc. However, it is obvious that the quality of the predictions would be highly dependent on the nature of the stored historical process data.

In 2010, Chitralekha and Shah [67] reported a steadystate soft sensor (based on a SVR approach) to estimate the material's MFI of an industrial polymer extrusion plant. They stated that the SVR-based soft sensor outperformed a existing nonlinear least-square-based soft sensor in terms of lower prediction errors while performing well over a wide range of melt indices. Moreover, they claimed that their soft sensor has a few tuning parameters only (compared to the existing neural network based soft sensors) and also showed good generalization capabilities over other nonlinear black-box modelling approaches.

Tian et al [68] proposed a MFI soft sensor based on an improved orthogonal least squares (IOLS) algorithm for a nonlinear PP process. An orthogonal signal correction was applied to minimize the effects of noise which was uncorrelated with process output variables. The sensor was tested with 100 unseen data sets and outperformed the results obtained from OLS and PLS methods, based on the root mean squared error).

Work by Maki et al [69] attempted to use a linear model predictive controller incorporating a soft sensor for the quality control of a polyolefin process. MFI was used as the major measure of the product quality. A two-stage model (a combination of a first principle model and a regression model incorporated with a number of statistical techniques: partial least squares regression (PLS), multiple linear regression (MLR), principal components regression (PCR), radial basis function (RBF) neural networks, multi quadratic (MQ) and reciprocal multi quadratic (RMQ)) was used on this purpose but the estimations of the soft sensor were not satisfactory and hence were not able to achieve the expected specifications. One of the possible reasons for this poor performance may be the use of first principle models which mostly represent the ideal steady-state conditions but not the actual process dynamics.

Zhang and Liu [70] introduced a MFI soft sensor for an industrial PP polymerization process and this sensor was on a combination of an adaptive fuzzy neural network (A-FNN) and SVR (A-FNNSVR model). A-FNN was used to generate 
fuzzy rules while the SVR method was adopted to optimize the parameters associated with the soft sensor. A five-layer network was used with IF-THEN rules but there is no clear mention on the criteria which was considered in determining the rule-base. A-FNNSVR model estimated the MFI with a mean relative error of $0.51 \%$ on a set of unseen data.

Liu et al [71] proposed a just-in-time sequential least squares support vector regression (JS-LSSVR) nonlinear model to predict the product quality. The model was tested by predicting the MFI of an industrial polymerization plant where it has outperformed over five other soft prediction methods by providing the lowest relative prediction error. Another work by Liu and Chen [72] used an integrated just-in-time LSSVR (IJLSSVR) model on the same purpose and it was better than the existing LSSVR and just-in-time LSSVR (JLSSVR) models.

Shang et al [73] argued that the typical data-driven soft sensors are commonly attributed with steady-state conditions. Hence, such sensors may suffer from transient inaccuracies and poor robustness as operating with dynamic processes. Then, they proposed a dynamic soft-sensing approach incorporating finite impulse response and support vector machine algorithms to describe dynamic and nonlinear static relationships. The sensor was able to predict the MFI of PP (inside a depolymerization reactor) with a RMSE of less than 0.4. Also, it outperformed the conventional static models in terms of the dynamic accuracy and the practical applicability.

Study reported by Fan et al [74] proposed a MFI soft sensor based on a process state detection and an impulses response template (IRT) where the model parameters were identified by using particle swarm optimization (PSO) and simulated Annealing (SA) algorithms. Three different algorithms (PSO, SA and PSO-SA) were tested on an unseen data set obtained from a PP plant and achieved RMSE values of 0.0953, 0.0656 and 0.0570 , respectively.

Cheng and Li [75] proposed a MFI soft sensor as a measure of the product quality of an industrial PP polymerization plant. The sensor's model was implemented with a systematic least squares support vector machine (Sys-LS-SVM), and then a nonlinear isometric feature mapping method and a particle swarm optimization (PSO) algorithm were structurally integrated into the proposed model to search for the optimal values of the relevant parameters. The authors claimed that the soft sensor performed well and it can be used with the similar type of industrial processes as it is a generalized method.

Farsang et al [76] argued that the MFI is an important parameter to the polymerization industry in determining the product quality. Hence, they developed empirical process models based on a PCA approach to estimate the MFI of PP in an industrial reactor. A static and a dynamic PCA models were developed and the static model showed good accuracy but did not account the time dependency. They claimed that both models gave RMSE values less than $10 \%$ but nothing was mentioned on the possibility of the sensor's practical use.

Zhang et al [77] proposed a MFI soft sensor (incorporating nine key process variables) for an industrial PP plant based on an extreme learning machine (ELM) and a modified gravitational search algorithm (MGSA). The proposed MGSAELM model was able to predict the MFI with a mean relative error (MRE) of $0.72 \%$ on unseen data, compared with $1.31 \%$ and $1.70 \%$ MRE values achieved with an adaptive PSO-ELM model and a GSA-ELM model, respectively. The authors expect to develop a web-server for this prediction approach.

Few other previous works [78]-[82] also attempted in inferential prediction of MFI in polymer processing related applications. Particularly, a considerable number of attempts have been reported on MFI soft sensors for polymerization processes. MFI is one of the key quality indicators of the polymerization processes [83] and hence there might be a great interest for on-line MFI prediction as it cannot be measured directly with a physical sensor in real-time. Interestingly, lots of different computational approaches (PLS, PCA, RBF, SVR, OLS, MLR, RMQ, FNN, IRT, PSO, SA and ELM) have already been attempted in developing MFI soft sensors and these previous studies will provide a good platform for adapting these approaches to predict other important process parameters as well.

\section{Soft sensing of screw torque or screw load torque}

According to some previous work [84], [85], screw load torque (i.e., a part of the equivalent motor torque passed to the extruder screw) dynamics seem to provide useful information on the process mechanism inside an extruder barrel. As an integral measurement of the polymer state along the length of the extruder, it also has the potential to give an earlier and more sensitive indication of process instabilities than measurements at the die. Therefore, real-time monitoring of the screw load torque dynamics (i.e., torque changes due to process disturbances) and analyzing of the causes behind these variations may be useful in troubleshooting and also to further understand the process mechanisms.

In 2011, the author [86], [87] explored the possibility of predicting the process thermal stability inferentially in a single screw extrusion process. The melt pressure, screw load torque, and melt temperature were measured experimentally and then the possible correlations between fluctuations/dynamics of these signals were examined. However, the results did not indicate strong correlations between these signals. Here, it can be argued that the screw load torque signal is not sensitive enough to identify unstable melting issues as it is dominated by the torque associated to the solids conveying zone of the screw. However, melt temperature and melt pressure fluctuations showed slight correlations in their fluctuations particularly at low screw rotational speeds. Nevertheless, none of these signals showed sufficiently good performance for them to be used as a powerful tool of monitoring the process thermal stability inferentially.

Eker et al. [85] used a $25 \mathrm{~mm}$ diameter twin screw extruder which displayed an estimation of the total screw torque based on the motor armature current. Then, they proposed a model to estimate the screw torque as a function of mass throughput, screw speed, material holdup constants and material compositions of partially and fully filled sections of a blending 
process with controlled feed rates. The machine displayed and the model estimated screw torque values were compared to prove the validity of the proposed model and achieved an overall $93.4 \%$ fit between these values. The difference between the estimated and measured torques were used for fault detection purposes. The authors argued that the screw torque/power can reflect the high frequency changes in the melt properties and throughput. Moreover, an observer method was proposed to estimate extruder screw torque from motor electrical variables as the torque signals provided by extruder control electronics were highly affected by noise. The performance of the observer was verified through simulation. However, the method proposed only applies to induction motors (i.e. a type of alternating current (AC) motor). In fact, the majority of the industrial extruders use direct current (DC) motors even though a.c. motors are sometimes found on some of the modern extruders [88].

Wassik and Camp [89] attempted to maintain the torque level of an extruder but did not try to examine the possible causes behind torque fluctuations. They claimed that the screw torque fluctuations cause fluctuations in melt temperature and pressure, and hence affect the product quality. Also, they argued that the torque on the screw is directly influenced by the size of the melt pool. Furthermore, they proposed an extruder model based on balancing the input and output materials and this model was used to develop a controller to maintain a constant screw torque during the process by manipulating the screw speed. Buja et al. [90] proposed three different methods to estimate the motor disturbance torque of a sensorless DC motor by extending the classical observer theory. The aim of this study was to make the drive motor more robust against load changes and unmodeled torques by knowing the actual impact, imposed by the disturbance torque, on the motor. However, the simulation results showed that only one scheme was able to estimate the disturbance torque accurately.

It should be noted that all fluctuations apparent in torque signals may not be associated with the process. There can be some other factors (e.g. screw vibrations, issues of speed transmission system or motor speed control) which may affect the level and fluctuations of the screw torque. Sun et al. [91] observed the screw vibration behaviour under different processing conditions using an ultrasonic test. Four ultrasound transducers were mounted on an extruder barrel close to the screw tip at $90^{\circ}$ intervals, orthogonally to the screw surface. Ultrasonic pulses were sent at a particular rate and echoes reflected by the rotating screw were captured. The gap between the screw surface and transducer was measured based on the signals' reflection times and the speed of sound. They found that the screw vibration behaviour was depended on how the screw was engaged into the gearbox sleeve and the status of the gearbox. In fact, such vibrations associated with an extruder screw may contribute to motor power/torque fluctuations.

\section{E. Soft sensing of other parameters}

Apart from the die melt temperature profile, melt viscosity, MFI and torque; a few attempts have been made to inferen- tially predict some other parameters in polymer processing

Weissman et al [92] proposed an inferential approach to predict the required energy to mould a part in injection moulding (i.e., before the part has gone into the production). The prediction algorithm includes five steps and works with a limited amount of data available during the design stage: a $\mathrm{CAD}$ model of the part, the material name, and the production requirements. Although the complete estimation procedure has been presented, the model has not been tested so far.

In the production of various grades of polymers, the predictive accuracy of process parameters deteriorates during the grade transition as the state of polymer reactors is quite unsteady, causing the values of process variables to differ from the steady-state values. Hence, Kaneko et al [93] introduced some models that can detect the completion of transition of polymer grades in order to reduce the amount of off-grade material in such various grade polymer plants. In this study, four types of models were developed: a k-nearest neighbour algorithm (k-NN), a support vector machine (SVM), a rangebased approach (RANGE) and a one-class SVM (OCSVM). A combination of a OCSVM and a PLS model gave the best accuracy and prediction rates. They claimed that their method could be applied for industrial polymer plants and also for other time series processes.

Work by Wang et al. [94] proposed a real-time soft sensing approach, incorporating infrared spectroscopy and ultrasonic velocity measurements, to estimate the degree of orientation of polymeric materials. A SVM approach was used to map a relationship between the ultrasonic velocity and the degree of orientation. The proposed inferential approach made accurate measurements only with a relative error of around $5 \%$.

Noor and Ahmad [95] developed a soft approach to predict the molecular weight of biopolymers using a neural networks. The proposed model correlated the various reaction temperatures of biopolymerization process with biopolymer's molecular weight. The simulation results showed good performance by predicting the molecular weight only with a sum squared error of 0.0926 on an unseen data. Further development to this approach would be really useful as the molecular weight is a parameter that cannot be physically measured in real-time.

Keneko and Funatsu [96] used a genetic algorithm-based wavelength selection (GAWLS) and a SVR based model to predict the polymer quality of an industrial reactor. Around 20 variables (including melt flow rate, pressure and temperature) were considered in predicting polymer quality and the GAWLS-SVR model outperformed the techniques such as PLS and GAWLS. They argued that this method would be really useful when a large number of variables have to be considered for ensuring the process functionality and product quality.

Souza and Arajo [97] proposed univariate linear regression models for adaptive soft sensing and those were used to predict the catalyst activity in the multi-tube of a polymerization plant. The results were better than the existing methods: a recursive least squares (RLS), a recursive partial least squares (RPLS), an incremental local learning soft sensing algorithm (ILLSA) and an online sequential extreme learning machine (OS-ELM). 
Cui at al [98] introduced soft sensing models, based on a echo state network (ESN), for real-time estimation of the conversion rate of vinyl chloride monomer in a polyvinyl chloride (PVC) polymerization process. A kernel principal component analysis (KPCA) approach was used in data processing where 10 input variables were considered. Then, a biogeographybased optimization algorithm (BBOA) was employed to optimize the structural parameters of the ESN to realize the nonlinear mapping between input and output parameters of the models. Eventually, three models were compared (ESN, BBOESN and BBO-Migration ESN) and achieved RMSE values of $0.0880,0.0856$ and 0.0713 , respectively. This work is based only on simulation and the authors claimed that the proposed models can significantly improve the prediction precision of conversion rate and velocity in PVC polymerization and also can satisfy the real-time control requirements of the process.

Shardt et al [99] came-up with a process monitoring approach (incorporated with a soft sensor) which can efficiently detect the process faults even in the absence of direct process information. As they argued, in process monitoring and fault diagnosis using both model-based and data-driven approaches, the information on key performance indicators (KPIs) such as concentration or density (which may need to be analyzed in a laboratory) may not be available at every sampling interval or immediately after sampling, in complex chemical or metallurgical processes. For such processes, the proposed data-driven diagnostic-observer-based soft sensor should be useful in detecting changes given in infrequently measured KPIs. The proposed scheme was validated statistically using a Monte Carlo simulation and practically applied to a TennesseeEastman process. They observed that increasing the sampling time or delay decreases the amount of information available about the process (as was expected), however the proposed soft sensor was able to detect the faults without too much of a delay given the loss of information. Moreover, the use of a filter was recommended to avoid high-frequency noise issues.

Taylor et al. [100] reported an inferential system to monitor the cross-section of plastic products formed in an extrusion process. A sensing system used a laser beam reflected from the surface which was capable of detecting the position and orientation of any desired point on the surface. The measurement precision of the profilometer was better than $10 / \mathrm{spl} \mathrm{mu} / \mathrm{m}$.

Work by Fan et al [101] proposed a wireless sensor for real-time monitoring of melt temperature and melt pressure inside a mould cavity of an injection moulding machine. The sensor was self-energized via electrical pluses generated based on the mould pressure. The proposed sensor's readings were well-matched with a commercially available wired sensor with mismatch errors of less than $\pm 5 \%$.

Peng et al [102] introduced a online visualization technique to observe the cell nucleation and evolution behavior in an extrusion die of a polymer foaming process. However, this system has a specifically developed slit die to facilitate the visualization and hence will not yet be suitable for industrial use. Additionally, a few other works also attempted to inferentially predict some other process parameters (particularly relating to the product quality) in polymer processing [103][111] or proposed advanced soft sensing techniques for other industrial processes [10], [112], [113].

\section{DISCUSSION}

Overall, the reported soft sensing approaches are mostly based on polymerization or extrusion processes. Perhaps this may be due to the polymerization is one of the main processes in the production and processing of polymers and also extrusion is served as an intermediate/initial processing step in several other processing techniques such as blown film, blow moulding, thermo-forming, injection moulding, extrusion coating/tubing, coextrusion and so forth. Additionally, soft sensing attempts were reported with injection moulding and compounding processes as well. A summary of the reported major soft sensing approaches in polymer processing (discussed above) is given in Table 1.

Generally, implementation of a soft sensor is a kind of complex task as the designer should have a sound knowledge on several aspects such as process operation/mechanisms, system identification and computer programming while all of these tasks should be performed carefully (mostly manually) to develop an appropriately tuned sensor. Some of the common challenging tasks in designing soft sensors are:

- Identification of key process variables

- Collection of an accurate data set form system identification experiments (with no missing or drifting data, outliers, measurement delays/noise, co-linear features and varying sampling rates, etc.)

- Selection of an appropriate modelling technique

- Development of generic/adaptive dynamic process models which are compatible with real-time applications to replicate the process with an acceptable accuracy

- Implementation of the soft sensor efficiently and accurately with computer programming tools (without demanding high performance computing, etc)

- Tuning of the sensor and long-term maintenance

Currently, numerous advanced techniques are being used in the industry/research to overcome these challenges. However, further improvements should be invaluable for developing reliable and accurate soft sensors. In polymer processing, the commonly used point/bulk measurement sensors are not quite good for diagnosing actual information on the process thermal stability. Therefore, monitoring of a temperature profile across the melt flow would be invaluable for determining the actual melt quality and also for developing advanced thermal control strategies. Moreover, the real-time physical measurements of parameters such as melt viscosity and melt flow index are also still challenging in industry although a number of soft sensing techniques have been proposed to predict these parameters in research. Clearly, there is an issue in the present polymer processing sector due to the lack of well-established techniques for making real-time measurements of key process parameters which are really important in process monitoring/control and hence in achieving the highest possible product quality. As was discussed, soft sensing approaches would be the most 
Table I

SUMMARY OF THE EXISTING MAJOR CONTROL APPROACHES FOR CONTINUOUS SINGLE SCREW EXTRUSION

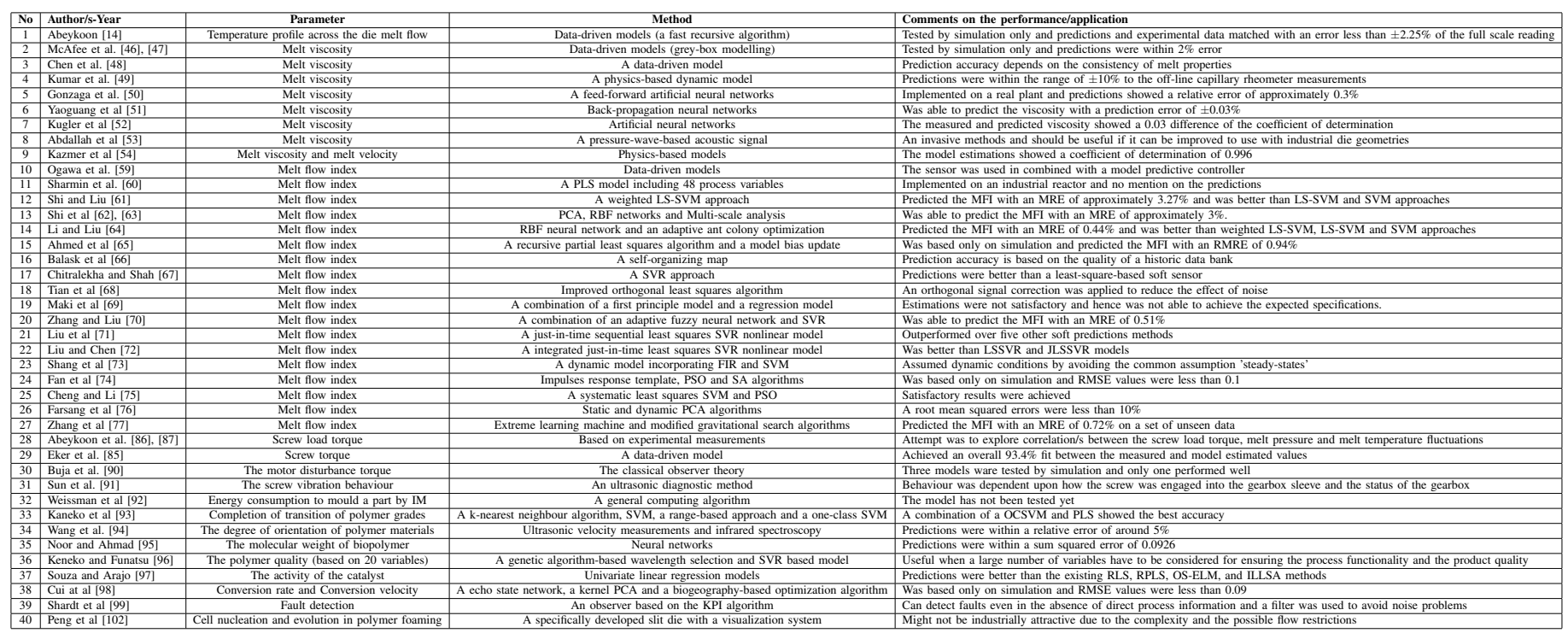

appropriate solution(s) for the majority of these measurements due to the complexities attributed of using physical sensors.

\section{A. Possible future directions and challenges}

The main function of soft sensors is to expand the process monitoring capabilities to collect more information about the process. These can be used as a continuous source of information which should be useful to improve the process control performance (can be used with automated control loops as well) and hence should assist in achieving better product quality [4]. Also this information can guide/assist process operators in decision making on the selection of process setting and functionality. Obviously the soft sensing is an emerging application in a number of industrial sectors with the advancements of computational facilities over the last couple of decades. Since soft sensing is mostly used when physical sensing is difficult or unavailable it will remain challenging due to the possible constraints specific to each application.

One of the common current trends (and also in the future as well) particularly in process industry is to use data-driven (or empirical) models of the process to predict the parameters that are practically difficult to measure in real-time. Here, the development of accurate and reliable process models is really important and is one of the main challenges. Once the models are available, sensing is quite straight forward and also can be implemented quite easily in most of the cases. However, the models may not be easily adapted to different machines and materials which will be another major concern. For example, for a polymer processing company with ten machines and who processes a large number of materials might have to develop/validate at least ten models which can be costly and time consuming. The development of a generalized (or an adaptive) model will be a possible solution but might not get the same accuracy with all the machines/materials. However, the data-driven models may be good in replicating actual process dynamics compared to the first principle models.

The use of physics-based models or known as the first principle models) would also be possible but would not be attractive for most of the future industrial applications due to their complexities/limitations in using real-time applications (e.g., difficulty of achieving closed-form solutions, simplifying assumptions). However, these may be an option for some of the simple processes that can be accurately represented by physics-based models (for the processes which are not deviating considerably form the steady-state conditions in practice). Nevertheless, it would be really useful in many aspects if one can develop first principle model(s) of their industrial processes as these models can be used to explore the chemical and physical background of the process where necessary.

Also, the use of combined approaches of empirical and/or physics-based models together with physical sensors (for feedback) would be another possibility. Some of the recent work [14], [47] have used such systems where a physical sensor was used as an indirect feedback (i.e., to monitor a directly measurable parameter) to confirm the accuracy of the parameter/s predicted by a soft sensor.

Web-based sensors (i.e., sensor networks that can be monitored and controlled remotely via web applications [114]) are also another emerging area in the current industry due to their advantages such as easy access from a long distance, easiness of control/maintenance, flexible configuration, less use of wiring circuits, etc. In such systems, soft sensing might have vital roles to play as the whole world is moving towards 'internet-based' and 'wireless' approaches.

From the above information, it is obvious that the majority 
of the soft sensing techniques are based on data-driven dynamic models of the processes. However, such sensors may not always function/perform well as a black-box model might not be valid when a process is operated outside certain conditions where operation data used for modelling were obtained [115]. Thus, it is important to evaluate the validity of the soft sensor's output on-line by checking whether the estimation error exceeds its allowable limit when a measurement becomes available. In the long-term use, the drifts of the process operation might cause problem on the performance of the soft sensor and hence it should be compensated either by adapting or re-developing the model/s [106]. Chen et al [116] proposed a Kalman filter based model mismatch index method to evaluate the predictive performance of soft sensor models and to gather information on when to update these models. If there is any mismatch, models can be updated through a PLS regression with the guidance of an off-line training. Saptoro [117] also proposed an adaptive soft sensing technique which can adapt to process changes based on a just-in-time modelling approach. Recent work by Yao and Ge [118] proposed a moving window approach to update the soft sensor model (based on the saved recent window data sets) when there is a lack of process information. Such approach should be useful for highly variable/unpredictable processes. A few other work also proposed techniques in estimating the prediction accuracy of soft sensors [119]-[121]. Implementation of these types of supportive techniques in-line with soft sensors would be highly useful as it is quite difficult to guess when and how to update a soft sensor's model(s) by following the usual procedures.

As some soft sensors use data-driven models for their predictions, the accuracy of these models are obviously dependent upon the quality of the experimental data used for their testing and validation. Hence, the collection of good quality data is one of the primary challenges in designing a soft sensor. If these initial measurements were poor in accuracy, the resulting models would also be poor in accuracy. Also, the majority of soft sensors require real-time process measurements of readily measurable process parameters (from physical sensors) as inputs of the process models attributed to them. Hence, the reliability and accuracy of the predictions made by a soft sensor are also directly associated to the accuracy/quality of the real-time physical measurements of process parameters (in both of their design and operational stages). Therefore, the use of the best possible equipment (sensors and other DAQ devices) for process measurements is a crucial requirement for achieving good performance from a soft sensor. Additionally, all of the supportive physical sensors and DAQ devices should be calibrated properly (in timely basis as recommended by the manufacturer) while avoiding/minimizing possible offsets of their readings. Essentially, appropriate methods should be employed to explore the signal noise associated with process measurements and resulting adverse effects on a soft sensor's performance. As was reported in some of the previous works [99], [122], [123], filtering of the measured process signals should not be carried out just to avoid noise related issues or large fluctuations in these signals, to make the sensor/controller design process easier. This may cause filtering of the fluctuations which might be generated due to the actual processing instabilities, and hence the soft sensor may not be capable of estimating some real fluctuations attributed to the process which may really affect the process functionality. Therefore, it would be best to make the process measurements (i.e., measurements such as the barrel set temperatures and screw speed) to the highest possible accuracy by using good quality physical sensors and DAQ devices. If it is certain that some of the information captured by a measured signal is not relevant to the actual processing behaviour, then such signals can be filtered-out by using a properly designed filtering technique. Perhaps, some industrial processes may find that it is difficult to follow some advanced or specific procedures that can be employed in an experimental settings to make accurate measurements. Nevertheless, it would be better to check the functionality and accuracy of the hardware sensors (which are associated to a soft sensor) from time to time while visually observing the key process parameters on a screen in real-time. Obviously, such real-time monitoring should allow identifying the accuracy of these measurements while observing the process functionality and health.

Once the data is recorded using a well-tuned DAQ unit with an appropriate sampling frequency, then the data processing is also a challenge as it is important to check the data sets for possible outliers, missing data points, offsets, etc. The selection of the most appropriate model structure and modelling technique is also vital, and the practical and theoretical knowledge on the process operation/mechanisms would be really useful on this aspect. The model optimization should also be performed to select the most efficient model (e.g., size of the model, required computational power, prediction accuracy) to be used in the soft sensor. Then, the implementation of the soft sensor should be performed carefully with a suitable software platform. As it is clear, a lots of different computational approaches (PLS, PCA, ANN, RBF, SVR, OLS, MLR, RMQ, FNN, IRT, PSO, SA and ELM) have been used in the soft sensor development. However, it should be noted that each of these methods has advantages, drawbacks and limitations specific to each of them. For example, PCA and PLS are type of powerful and popular computational techniques but pure PCA/PLS can only effectively handle linear relationships and may not perform well with the nonlinearity of the data. One of the possible problems with artificial neural networks (ANN) is that it can get stuck in local minima during the learning process. SVM training process may be computationally complex when dealing with very large data sets. Evidently, some previous works have used improved/combined algorithms to avoid some of these limitations of individual techniques. Therefore, it is essential that the designer should have a sound knowledge on these types of complexities and limitations as well. Kadlec et al [2] have provided a detailed discussion on the design and application of data-driven soft sensors in the process industry. A few other previous works [5], [6], [20], [124]-[126] also 
discussed some issues/challenges relating to the design and application of soft sensors and can be found in the literature.

At present, most of the tasks related to soft sensor design (e.g., data processing, model selection/validation, sensor maintenance, adaptation, (see Fig. 1)) are still preformed manually. Hence, as was highlighted, the performance/quality of soft sensors are highly dependent upon the designer's knowledge and experience and also may have high likeliness of making mistakes while consuming lots of time. In future, it would be good to explore the possibility of making these tasks to be automated fully or partially to improve the accuracy and reliability. Hence, more research is recommended in developing adaptive soft sensors associated with automatic adaptation features.

Clearly, the majority of proposed soft sensing techniques have been tested only with simulations and are not yet ready for practical use. Therefore, soft sensing aspects relating to polymer processing are also still considerably under-developed and hence further researches are highly recommended. Improving of soft sensors from simulation stage to practical use would be challenging as the actual process dynamics can really be complicated than the conditions considered in simulation experiments. Usually, polymer processing techniques are highly complex in nature particularly due to the coupled nature of the key process variables/parameters [24], [25]. Hence, it seems that this is the best time for researchers to think about developing practically viable adaptive soft sensing approaches by moving one step ahead from the simulation. Such soft sensors would be really useful for the improved process monitoring and control of polymer processing, and also for other industrial applications as well.

\section{CONCLUSIONS AND FUTURE WORK}

In general, soft sensing techniques are becoming increasingly popular in wide range of industrial applications. Today, advanced mechatronics and computing techniques provide an ideal platform for developing attractive and accurate soft sensing strategies for process monitoring, fault diagnostics, process control, and so forth. Such techniques are very useful to apply when physical sensors cannot be employed or not available for direct measurements. In case of designing/using soft sensing techniques, it is quite important to follow the steps given in Figure 1 with proper procedures and also these sensors should be properly maintained in the long-term use. It is well-known that the polymer processing techniques are quite difficult to monitor directly in real-time as materials' processing usually occur inside a closed chamber/barrel. Therefore, a number of researchers attempted to use soft sensing approaches to predict parameters such as melt viscosity, melt flow index, melt temperature and screw load torque and so forth. Obviously, soft sensing and soft sensors are hot topics in the present manufacturing industry where lots of research and development activities are underway. However, the majority of the existing or on-going work are limited to simulation experiments and only a few attempts have been reported on using soft sensors in real industrial/practical applications. Hence, it is really important to extend the research and development for developing industrially compatible soft sensors for the betterment of future of the manufacturing industry. Obviously, we cannot undermine the usefulness of hardware/physical sensors as these will be used in collecting information for the functionality of most the soft sensing techniques as well. Given the prevalent conditions, it can be predicted that future world would be interested in soft, clean, adjustable, reliable and advanced techniques which would be ideally based on soft platforms (in top layer) but with the help of several hardware/physical sensors in the bottom layer.

\section{REFERENCES}

[1] K. R. Foster, "Software tools," IEEE Spectrum, vol. 35, no. 1, pp. 52-56, 1998.

[2] P. Kadlec, B. Gabrys, and S. Strandtb, "Data-driven soft sensors in the process industry," Chem. Eng. Res. Des., no. 4, pp. 795-814, 2009.

[3] L. Fortuna, S. Graziani, A. Rizzo, and M. G. Xibilia, Soft Sensors for Monitoring and Control of Industrial Processes. Springer, 2006.

[4] N. T. Nguyen and R. Katarzyniak, New Challenges in Applied Intelligence Technologies, 1st ed. Springer, 2008.

[5] G. D. Gonzalez, "Soft sensors for processing plants," The 2nd Int. Conf. on Intelligent Processing and Manufacturing of Materials, Honolulu, USA, vol. 1, pp. 59-69, 1999.

[6] L. Fortuna, S. Graziani, and M. G. Xibilia, "Comparison of soft-sensor design methods for industrial plants using small data sets," IEEE Trans. Instrum. Meas., vol. 58, no. 8, pp. 2444-2451, 2009.

[7] P. Kadleca, R. Grbic, and B. Gabrys, "Review of adaptation mechanisms for data-driven soft sensors," Chem. Eng. Res. Des., vol. 35, no. 1 , pp. 1-24, 2011.

[8] C. Abeykoon, "Soft sensing of melt temperature in polymer extrusion," 15th European Control Conf., Aalborg, Denmark, pp. 340-345, 2016.

[9] F. A. A. Souza, R. Arajo, and J. Mendes, "Review of soft sensor methods for regression applications," Chemom. Intell. Lab. Syst., vol. 152, pp. 69-79, 2016.

[10] M. R. Serpas, "Soft sensors for process monitoring of complex processes," Ph.D. dissertation, Texas A and M University, USA, 2012.

[11] Z. X. Wanga, Q. P.Heb, and J. Wang, "Comparison of variable selection methods for PLS-based soft sensor modeling," J. Process Control, vol. 26, pp. 56-72, 2015.

[12] Y.-L. Hea, Z.-Q. Genga, and Q.-X. Zhua, "Data driven soft sensor development for complex chemical processes using extreme learning machine," Chem. Eng. Res. Des., vol. 102, pp. 1-11, 2015.

[13] C. Shanga, F. Yanga, D. Huanga, and W. Lyua, "Data-driven soft sensor development based on deep learning technique," J. Process Control, vol. 24, no. 3, pp. 223-233, 2014.

[14] C. Abeykoon, "A novel soft sensor for real-time monitoring of die melt temperature profile in polymer extrusion," IEEE Trans. Ind. Electron., vol. 61, no. 12, pp. 7113-7123, 2014.

[15] J. Deng, L. Xie, L. Chen, S. Khatibisepehr, B. Huang, F. Xu, and A. Espejo, "Development and industrial application of soft sensors with on-line bayesian model updating strategy," J. Process Control, vol. 23, no. 3, pp. 317-325, 2013.

[16] S. Khatibisepehr, B. Huang, and S. Khare, "Design of inferential sensors in the process industry: A review of bayesian methods," $J$. Process Control, vol. 23, no. 10, pp. 1575-1596, 2013.

[17] D. Slikovic, R. Grbic, and Z. Hocenski, "Methods for plant data-based process modeling in soft-sensor development," Automatika, vol. 52, no. 4, pp. 306-318, 2011.

[18] D. Wang, J. Liu, and R. Srinivasan, "Data-driven soft sensor approach for quality prediction in a refining process," IEEE Trans. Ind. Inf., vol. 6 , no. 1 , pp. 11-17, 2010.

[19] B. Lin, B. Recke, J. K. Knudsen, and S. B. Jrgensen, "A systematic approach for soft sensor development," Comput. Chem. Eng., vol. 31, no. 5-6, pp. 419-425, 2007.

[20] G. D. Gonzalez, J. P. Redard, R. Barrera, and M. Fernandez, "Issues in soft-sensor applications in industrial plants," IEEE Int. Symp.on Industrial Electronics, Santiago, USA, pp. 380-385, 1994.

[21] T. Sakai, "Screw extrusion technologypast, present and future," Polimery, vol. 58, no. 11-12, pp. 847-857, 2013. 
[22] PasticsEurope, "Plastics - the facts 2014/2015: An analysis of european plastics production, demand and waste data," 2013, available at: https :

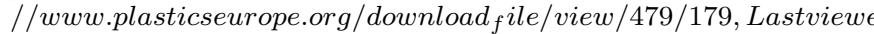
20 thof May2018.

[23] C. Holzer, "The future of polymer processing," Polimeri, vol. 32, no 3-4, pp. 124-129, 2011.

[24] Z. Tadmor and C. G. Gogos, Principles of Polymer Processing, 2nd ed. Wiley, 2006.

[25] T. A. Osswald, Understanding Polymer Processing: Processes and Governing Equations. Hanser-Munich, 2015.

[26] A. N. Wilkinson and A. J. Ryan, Polymer Processing and Structure Development. Kluwer Acadamic Publishers, 1999.

[27] C. Rauwendaal, Polymer extrusion. Hanser-Munich, 2001

[28] C. Abeykoon, Polymer Extrusion: A Study on Thermal Monitoring Techniques and Melting Issues. Lap Lambert Publishing:Verlag, 2012.

[29] M. J. Stevens and J. A. Covas, Extruder principles and operation, 2nd ed. Chapman \& Hall, 1995.

[30] C. Abeykoon, "Single screw extrusion control: A comprehensive review and directions for improvements," Control Eng. Pract., vol. 51, pp. 6980,2016

[31] J. M. Maia, "On-line sensoring in extrusion-based processes: past, present and future," SPE-ANTEC Tech. Papers, vol. 1, 2012.

[32] C. Abeykoon, "Modelling and control of melt tempearture in polymer extrusion,” Ph.D. dissertation, Queen's University Belfast, UK, 2011.

[33] C. Abeykoon, P. J. Martin, A. L. Kelly, and E. C. Brown, "An evaluation of melt temperature sensors for polymer extrusion," Sens. Actuators, A, vol. 182, pp. 16-27, 2012.

[34] X. Shen, R. Malloy, and J. Pacini, "An experimental evaluation of melt tempearture sensors for thermopalstic extrusion," SPE ANTEC Tech. Papers, pp. 918-926, 1992.

[35] V. Schoppner, K. Enns, K. Anger, and E. Giese, "Dynamic temperature measurement in polymer processing," SPE ANTEC Tech. Papers, vol. 1, pp. 228-232, 2008.

[36] A. J. Bur, M. G. Vangel, and S. C. Roth, "Fluorescence based temperature measurements and applications to real-time polymer processing," Polym. Eng. Sci., vol. 41, no. 8, pp. 1380-1389, 2001.

[37] E. C. Brown, A. L. Kelly, and P. D. Coates, "Melt temperature field measurement in single screw extrusion using thermocouple meshes," Rev. Sci. Instrum., vol. 75, no. 11, pp. 4742-4748, 2004.

[38] C. Abeykoon, "A novel model-based controller for polymer extrusion," IEEE Trans. Fuzzy Syst., vol. 22, no. 6, pp. 1413-1430, 2014.

[39] C. Abeykoon, K. Li, M. McAfee, P. J. Martin, Q. Niu, A. L. Kelly, and J. Deng, "A new model based approach for the prediction and optimisation of thermal homogeneity in single screw extrusion," Control Eng. Pract., vol. 19, no. 8, pp. 862-874, 2011

[40] H. K. Bruss, "Viscosity measurement for the automatic control and monitoring of the uniformity of extrusion processes," Kunststoffe German Plastics, vol. 60, no. 3, pp. 162-164, 1970.

[41] C. Rauwendaal and F. Fernandez, "Experimental study and analysis of a slit die viscometer," Polym. Eng. Sci., vol. 25, no. 12, pp. 765-771, 1985

42] J. M. Dealy and T. O. Broadhead, "Process rheometers for molten plastics: a survey of existing technology," Polym. Eng. Sci., vol. 33, no. 23, pp. 1513-1523, 1993.

[43] M. Padmanabhan and M. Bhattacharya, "In-line measurement of rheological properties of polymer melts," Rheol. Acta, vol. 33, no. 1, pp 71-87, 1994.

[44] S. H. Chiu, H. C. Yiu, and S. H. Pong, "Development of an in-line viscometer in an extrusion molding process," J. Appl. Polym. Sci. vol. 63, no. 7, pp. 919-924, 1997.

[45] S. H. Chiu and S. H. Pong, "In-line viscosity fuzzy control," J. Appl. Polym. Sci., vol. 79, no. 7, pp. 1249-1255, 2001

[46] M. McAfee, "A soft sensor for viscosity control of polymer extrusion," Ph.D. dissertation, Queen's University Belfast, UK, 2005.

[47] M. McAfee and G. McNally, "Real-time measurement of melt viscosity in single-screw extrusion," Trans. Inst. Meas. Control, vol. 28, no. 5, pp. 481-499, 2006

[48] Z. L. Chen, P. Y. Chao, and S. H. Chiu, "Proposal of an empirical viscosity model for quality controlling the polymer extrusion process,' Polym. Test., vol. 22, no. 5, pp. 601-607, 2003.

[49] A. Kumar, S. A. Eker, and P. K. Houpt, "A model based approach for estimation and control for polymer compounding," IEEE Conf. on Control Applications, vol. 1, pp. 729-735, 2003.
[50] J. Gonzaga, L. Meleiro, C. Kiang, and R. M. Filho, "ANN-based softsensor for real-time process monitoring and control of an industrial polymerization process," Comput. Chem. Eng., vol. 33, no. 1, pp. 4349, 2009.

[51] H. Yaoguang, C. Xi, C. Xiangmin, Z. Ruijun, and Y. Yan, "Soft sensor modelling of feed liquid viscosity control for pvc gloves based on bp neural network," Proc. of the 2010 IEEE IEEM, pp. 1438-1442, 2010.

[52] C. Kugler, K. Dietl, T. Hochrein, P. Heidemeyer, and M. Bastian, "Robust soft sensor based on an artificial neural network for real-time determination of the melt viscosity of polymers," Proc. of the Polymer Processing Society 29th Annual Meeting, July 15-19, Nuremberg, Germany, vol. 126, pp. 213-216, 2013.

[53] A. Abdallah, S. Clara, E. K. Reichel, G. Brabants, B. Jakoby, H.-J. Luger, I. Burzic, A. Lepschi, J. Miethlinger, T. Voglhuber-Brunmaier, and V. Putz, "Acoustic sensor for in-line monitoring in polymer extrusion dies," vol. 52, pp. 1-4, 2015.

[54] D. O. Kazmer, G. W. Gordon, G. A. Mendible, S. P. Johnston, X. Tang, Z. Fan, and R. X. Gao, "A multivariate sensor for intelligent polymer processing," IEEE/ASME Trans. Mechatron., vol. 20, no. 3, pp. 1015 1023, 2015.

[55] B. K. Nguyen, G. McNally, and A. Clarke, "Real time measurement and control of viscosity for extrusion processes using recycled materials," Polym. Degrad. Stab., vol. 102, pp. 769-778, 2007.

[56] S. H. Chiu and S. H. Pong, "In-line viscosity control in an extrusion process with a fuzzy gain scheduled pid controller," J. Appl. Polym. Sci., vol. 74, no. 3, pp. 541-555, 1999.

[57] S. H. Chiu and C. C. Lin, "Applying the constrained minimum variance control theory on in-line viscosity control in the extrusion moulding process," J. Polym. Res., vol. 5, no. 3, pp. 171-175, 1998.

[58] J. M. Dealy and K. Wissbrun, Melt Rheology and Its Role in Plastics Processing: Theory and Applications, 4th ed. Kluwer Academic Publishers, 1999

[59] M. Ogawa, M. Ohshima, K. Morinaga, and F. Watanabe, "Quality inferntial control of an industrial high density polyethylene process," J. Process Control, vol. 9, no. 1, pp. 51-59, 1999.

[60] R. Sharmin, U. Sundararaj, and Y.-J. S. S. Shah, L. V. Griend, "Inferential sensors for estimation of polymer quality parameters: Industrial application of a PLS-based soft sensor for a LDPE plant,' Chem. Eng. Sci., vol. 61, no. 19, pp. 6372-6384, 2006.

[61] J. Shi and X.-G. Liu, "Melt index prediction by weighted least squares support vector mechanics," J. Appl. Polym. Sci., vol. 101, no. 1, pp. $285-289,2006$

[62] C. Zhang, X. Liu, J. Shi, and J. Zhu, "Neural soft-sensor of product quality prediction," 6th World Congress on Intelligent Control and Automation, June 21 - 23, 2006, Dalian, China, pp. 4881-4885, 2006.

[63] J. Shi and X.-G. Liu, "Product quality prediction by a neural soft-sensor based on msa and pca," Int. J. Autom. Comput., vol. 1, pp. 17-22, 2006.

[64] J. Li and X.-G. Liu, "Melt index prediction by RBF neural network optimized with an adaptive new ant colony optimization algorithm," $J$ Appl. Polym. Sci., vol. 119, no. 5, pp. 3093-3100, 2010.

[65] F. Ahmed, S. Nazir, and Y. K. Y. Yeo, "A new soft sensor based on recursive partial least squares for online melt index predictions in grade-changing hdpe operations," Chem. Prod. Process Model., vol. 4 , no. 1, 2009, article 33, DOI: 10.2202/1934-2659.1271.

[66] B. Balask, S. Nmeth, and J. Abonyi, "Integrated process and control system model for product quality control - a soft-sensor based application," European Control Conf., Budapest, Hungary, August 2326, 2009, pp. 3498-3502, 2009

[67] S. B. Chitralekha and S. L. Shah, "Application of support vector regression for developing soft sensors for nonlinear processes," Can J. Chem. Eng., vol. 88, pp. 696-709, 2010.

[68] H. Tian, X. Tian, and X. Deng, "Soft sensor for polypropylene melt index based on improved orthogonal least squares," 8th World Congres. on Intelligent Control and Automation July 6-9 2010, Jinan, China, pp. 5881-5885, 2010

[69] T. Maki, A. Yamaguchi, and M. Shimomura, "The methods of soft sensor modeling for polyolefin quality," 8th SICE Annual Conf., August 20-23, 2012, Akita University, Akita, Japan, pp. 327-332, 2012.

[70] M. Zhang and X. Liu, "A soft sensor based on adaptive fuzzy neural network and support vector regression for industrial melt index prediction," Chemom. Intell. Lab. Syst., vol. 126, pp. 83-90, 2013.

[71] Y. Liu, Z. Gao, and J. Chen, "Development of soft-sensors for online quality prediction of sequential-reactor-multi-grade industrial processes," Chem. Eng. Sci., vol. 102, no. 11, pp. 602-612, 2013. 
[72] Y. Liu and J. Chen, "Integrated soft sensor using just-in-time support vector regression and probabilistic analysis for quality prediction of multi-grade processes," J. Process Control, vol. 23, no. 6, pp. 793804, 2013.

[73] C. Shang, X. Gao, F. Yang, and D. Huang, "Novel bayesian framework for dynamic soft sensor based on support vector machine with finite impulse response," IEEE Trans. Control Syst. Technol., vol. 22, no. 4, pp. 1550-1557, 2014

[74] Z. Fan, J. Cao, and Y. Wei, "Dynamic soft sensor modeling based on state detection and impulses response template," The 26th Chinese Control and Decision Conf., June 2014, Changsha, Chaina, pp. 40314037, 2014, 10.1109/CCDC.2014.6852886.

[75] Z. Cheng and X. Liu, "Optimal online soft sensor for product quality monitoring in propylene polymerization process," Neurocomputing, vol 149, no. C-3, pp. 1216-1224, 2015.

[76] B. Farsang, I. Balogh, S. Nmeth, Z. Szkvlgyi, and J. Abonyi, "Spca based data reconciliation in soft sensor development application for melt flow index estimation," Chem. Eng. Trans., vol. 43, pp. 1555$1560,2015$.

[77] M. Zhang, X. Liu, and Z. Zhang, "A soft sensor for industrial melt index prediction based on evolutionary extreme learning machine," Chin. J. Chem. Eng., 2016, DOI: 10.1016/j.cjche.2016.05.030.

[78] Z. Zhang, T. Wang, and X. Liu, "Melt index prediction by aggregated rbf neural net works trained with chaotictheory," Neurocomputing, vol 131, pp. 368-376, 2014.

[79] L. Xia and H. Pan, "Inferential estimation of polypropylene melt index using stacked neural networks based on absolute error criteria," Int. Conf. on Computer, Mechatronics, Control and Electronic Engineering, Aug 2010, Changchun, China, vol. 3, pp. 216-218, 2010.

[80] J. Liu, "On-line soft sensor for polyethylene process with multiple production grades," Control Eng. Pract., vol. 15, pp. 769-778, 2007.

[81] B. Feil, J. Abonyi, P. Pach, S. Nemeth, P. Arva, M. Nemeth, and G. Nagy, "Semi-mechanistic models for state-estimation soft sensor for polymer melt index prediction," Springer: Lecture Notes in Computer Science, vol. 3070, no. 17, pp. 1111-1117, 2004

[82] T. Yiagopoulos, C. U. Schmidt, and E. Maselli, "Development of a softsensor for on-line mfi monitoring in reactive polypropylene extrusion," Dech. Monog., vol. 138, pp. 305-309, 2004.

[83] W. J. Yoon, Y. S. Kim, I. S. Kim, and K. Y. Choi, "Recent advances in polymer reaction engineering: Modeling and control of polymer properties," Korean J. Chem. Eng., vol. 21, no. 1, pp. 147-167, 2004.

[84] S. J. Derezinski, "Dynamic torque of a single screw extruder," $S P E$ ANTEC Tech. Papers, vol. 1, 2001, paper No: 113.

[85] A. Eker, M. Giammattia, P. Houpt, A. Kumar, O. Montero, M. Shah, N. Silvi, and T. Cribbs, "Intelligent extruder for polymer compounding," GE Global Research, Automation \& Controls Lab (For U.S. Dept. of Energy, Contract DE-FC02-99-CH10972), Tech. Rep., 2002.

[86] C. Abeykoon, M. McAfee, K. Li, A. L. Kelly, and E. C. Brown, "Monitoring the effect of operating conditions on melt temperature homogeneity in single-screw extrusion," SPE ANTEC Tech. Papers, vol. 1, pp. 1799-1806, 2010.

[87] C. Abeykoon, M. McAfee, K. Li, P. J. Martin, and A. L. Kelly, "The inferential monitoring of screw load torque to predict process fluctuations in polymer extrusion," J. Mater. Process. Technol., vol 211, no. 12, pp. 1907-1918, 2011.

[88] H. F. Giles, J. R. Wagner, and E. M. Mount, "Extrusion - the definitive processing guide and handbook," William Andrew Publishing/Plastics Design Library, pp. 13-15, 2005.

[89] J. M. Wassick and D. T. Camp, "Internal model control of an industrial extruder," American Control Conf., Atlanta, USA, pp. 2347-2352, 1988

[90] G. S. Buja, R. Menis, and M. I. Valla, "Disturbance torque estimation in a sensor less dc drive," IEEE Trans. Ind. Electron., vol. 42, no. 4, pp. 351-357, 1995

[91] Z. Sun and L. A. Utracki, "Ultrasonic diagnosis of the vibration behaviour of screws in a single screw extruder," SPE ANTEC Tech. Papers, pp. 1935-1938, 2009.

[92] A. Weissman, S. K. Gupta, A. Ananthanarayanan, and R. D. Sriram, "A systematic methodology for accurate design-stage estimation of energy consumption for injection moulded parts," Int. Design Engineering Technical Conf. \& Computers and Information in Engineering Conf., August 2010, Montreal, Canada, 2010.

[93] H. Kaneko, M. Arakawa, and K. Funatsu, "Novel soft sensor method for detecting completion of transition in industrial polymer processes,' Comput. Chem. Eng., vol. 35, no. 6, pp. 1135-1142, 2011.
[94] Y. Wang, W. G. Lin, H. Xu, and T. Liu, "Study on measurement of polymer orientation degree base on SVM," Adv. Mater. Res., vol. 314 316, pp. 2389-2393, 2011.

[95] R. A. M. Noor and Z. Ahmad, "Neural network based soft sensor for prediction of biopolycaprolactone molecular weight using bootstrap neural network technique," The 3rd Conf. on Data Mining and Optimization, June 2011, Putrajaya, Malaysia, pp. 70-73, 2011.

[96] H. Kaneko and K. Funatsu, "Nonlinear regression method with variable region selection and application to soft sensors," Chemom. Intell. Lab. Syst., vol. 121, pp. 26-32, 2013.

[97] F. Souza and R. Arajo, "Online mixture of univariate linear regression models for adaptive soft sensors," IEEE Trans. Ind. Inf., vol. 10, no. 2, pp. 937-945, 2014

[98] W. hua Cui, J. sheng Wang, and S. xia Li, "Kpca-esn soft-sensor model of polymerization process optimized by biogeography-based optimization algorithm," Math. Probl. Eng., 2015, dOi:10.1155/2015/493248.

[99] Y. A. W. Shardt, H. Hao, and S. X. Ding, "A new soft-sensor-based process monitoring scheme incorporating infrequent kpi measurements," IEEE Trans. Ind. Electron., vol. 62, no. 6, pp. 3843-3851, 2015.

[100] R. Taylor, N. Hancock, and T. Tran-Cong, "Non-contact extrudate profilometer-introductory paper," 4th Conf. on Mechatronics \& Machine Vision in Practice, Queensland, Australia, pp. 158-162, 1997.

[101] Z. Fan, R. X. Gao, and D. O. Kazmer, "Design of a self-energized wireless sensor for simultaneous pressure and temperature measurement," IEEE/ASME Int. Conf. on Advanced Intelligent Mechatronics, Montreal,Canada, pp. 1168-1173, 2010.

[102] X. F. Peng, L. Y. Liu, B. Y. Chen, H. Y. Mi, and X. Jing, "A novel online visualization system for observing polymer extrusion foaming," Polym. Test., vol. 52, pp. 225-233, 2016.

[103] K. Mulrennana, J. Donovana, L. Creedona, I. Rogersb, J. G. Lyonsb, and M. McAfee, "A soft sensor for prediction of mechanical properties of extruded pla sheet using an instrumented slit die and machine learning algorithms," Polym. Test., vol. 69, pp. 462-469, 2018.

[104] E. Frauendorfer and W.Hergeth, "Soft sensor applications in industrial vinylacetate-ethylene (vae) polymerization processes," Macromol. React. Eng., vol. 11, no. 4, 2017.

[105] N. Kumar, O. Piccin, L. Meylheuc, L. Barb, and B. Bayle, "Design and modeling of a polymer force sensor," IEEE/ASME Trans. Mechatron., vol. 21 , no. 1, pp. 555-564, 2015.

[106] H. Kaneko and K. Funatsu, "Development of high predictive soft sensor method and the application to industrial polymer processes," Asia-Pac. J. Chem. Eng., vol. 7, no. S1, pp. S39-S47, 2012.

[107] P. Facco, F. Doplicher, F. Bezzo, and M. Barolo, "Moving average PLS soft sensor for online product quality estimation in an industria batch polymerization process," J. Process Control, vol. 19, no. 3, pp. 520-529, 2009

[108] V. Noeei, S. Zhu, C. Tzoganakis, T. Duever, D. Ross, R. Clarke, P. V. Laren, and R. Pop-hey, "Model-based inferential sensing of melt flow rate in polymer compounding operations," SPE ANTEC Tech. Papers, vol. 1, pp. 187-191, 2009.

[109] R. Rallo, J. Ferre-Gine, A. Arenas, and F. Giralt, "Neural virtual sensor for the inferential prediction of product quality from process variables," Comput. Chem. Eng., vol. 26, pp. 1736-1754, 2002.

[110] M. Ohshima and M. Tanigaki, "Quality control of polymer production processes," J. Process Control, vol. 10, pp. 135-148, 2000.

[111] J. Zhang, A. J. Morris, E. B. Martin, and C. Kiparissides, "Prediction of polymer quality in batch polymerisation reactors using robust neural networks," J. Appl. Polym. Sci., vol. 69, no. 2, pp. 135-143, 1998.

[112] F. Xu, Y. Wang, and X. Luo, "An adaptive soft sensor based on nonlinear differential-algebraic observer for chemical processes," IEEE Sensors J, vol. 15, no. 6, pp. 3249-3257, 2015.

[113] X. Yuan, Z. Ge, Z. Song, Y. Wang, C. Yang, and H.Zhang, "Soft sensor modeling of nonlinear industrial processes based on weighted probabilistic projection regression," IEEE Trans. Instrum. Meas., vol. 66 , no. 4, pp. 837-845, 2017.

[114] T. Fukatsu, T. Kiura, and M. Hirafuji, "A web-based sensor network system with distributed data processing approach via web application," Comput. Stand. Inter., vol. 33, pp. 565-573, 2011.

[115] M. Kano and M. Ogawa, "The state of the art in advanced chemical process control in japan: Good practice and questionnaire survey," $J$. Process Control, vol. 20, no. 9, pp. 969-982, 2010.

[116] K. Chen, I. Castillo, L. H. Chiang, and J. Yu, "Soft sensor model maintenance: A case study in industrial processes," 9th IFAC Sym. on 
Advanced Control of Chemical Processes, Whistler, Canada, vol. 48, no. 8, pp. 427-432, 2015.

[117] A. Saptoro, "State of the art in the development of adaptive soft sensors based on just-in-time models," Procedia Chemistry, vol. 9, pp. 226234, 2014.

[118] L. Yao and Z. Ge, "Online updating soft sensor modeling and industrial application based on selectively integrated moving window approach," IEEE Trans. Instrum. Meas., vol. 66, no. 8, pp. 1985-1993, 2017.

[119] H. Kaneko and K. Funatsu, "A soft sensor method based on values predicted from multiple intervals of time difference for improvement and estimation of prediction accuracy," Chemometr. Intell. Lab, vol. 109, no. 2, pp. 197-206, 2011.

[120] H. Kaneko and K. Funatsu, "Applicability domain of soft sensor models based on one-class support vector machine," AIChE J., vol. 59, no. 6, pp. 2046-2050, 2013.

[121] A. Abusnina, D. Kudenko, and R. Roth, "Selection of covariance functions in gaussian process-based soft sensors," IEEE Int. Conf. on Ind. Techno., Feb-Mar. 2014, Busan, South Korea, pp. 371-378, 2014.

[122] W. Fontaine, Ph.D. dissertation, Ohio State University, Ohio, 1975.

[123] M. H. Costin, P. A. Taylor, and J. D. Wright, "On the dynamics and control of a plasticating extruder," Polym. Eng. Sci., vol. 22, no. 17, pp. 1095-1106, 1982.

[124] C. Alippi, S. Ferrari, V. Piuri, M. Sami, and F. Scotti, "New trends in intelligent system design for embedded and measurement applications," IEEE Instrum. Meas. Mag., vol. 2, no. 2, pp. 36-44, 1999.

[125] H. J. G. Escobar, "Advanced monitoring and soft sensor development with application to industrial processes," Ph.D. dissertation, Auburn University, USA, 2012.

[126] W. Yan, P. Guo, Y. Tian, and J. Gao, "A framework and modeling method of data-driven soft sensors based on semisupervised gaussian regression,” Ind. Eng. Chem. Res., vol. 55, pp. 7394-7401, 2016.

Chamil Abeykoon is an Assistant Professor of the School of Materials, Faculty of Science and Engineering of the University of Manchester (UoM). Chamil received his BSc (Hons) in Mechanical Engineering from the University of Peradeniya, Sri Lanka with the award of best performance in Mechanical Engineering in 2007. Then, he received his $\mathrm{PhD}$ in Mechanical Engineering in 2011 from the Queens University Belfast, UK with several publications where one of his publications was awarded with a young author best paper award from the IEEE in 2011. After completing the $\mathrm{PhD}$ degree, he briefly worked as a Lecturer in Mechanical Engineering at the University of Peradeniya (Sri Lanka), as a Research Fellow at the University of Bradford (UK) and as a Lecturer in Engineering at the Glyndwr University (UK), before joining the UoM. Currently Chamil is working mainly with the Northwest Composts Centre and the Aerospace Research Institute, and supervising research students affiliated with both the School of Materials and the School of Mechanical and Aerospace Engineering at the UoM. So far, he has authored 40+ peer-reviewed journal/conference papers. Moreover, he is the author of the monograph Polymer Extrusion: A Study on Thermal Monitoring Techniques and Melting Issues. His current research interests include: Process Monitoring, Modelling and Control; Soft Sensors and Soft Sensing; Process Instrumentation; Renewable Energy Technologies; and Heat Transfer. He has served in scientific committees and also has been invited for keynote speeches of several international conferences. He is an associate member of the professional bodies IESL, IMechE and ICPM; and also a Fellow of the Higher Education Academy UK. Moreover, Chamil is an Associate Editor of the Journal of Fluid Flow, Heat and Mass Transfer. 